\title{
Вакуумное двулучепреломление в поле плоской электромагнитной волны
}

\author{
(C) И.А. Александров ${ }^{1,2}$, В.М. Шабаев ${ }^{1}$ \\ ${ }^{1}$ Санкт-Петербургский государственный университет, \\ 199034 Санкт-Петербург, Россия \\ ${ }^{2}$ ФТИ им. А.Ф. Иоффре, \\ 194021 Санкт-Петербург, Россия \\ e-mail: i.aleksandrov@spbu.ru
}

Поступила в редакцию 20.03.2021 г.

В окончательной редакции 31.03.2021 г.

Принята к публикации 02.04.2021 г.

Исследован процесс вакуумного двулучепреломления в сильном электромагнитном поле: пробный фотон изменяет свою поляризацию при прохождении через встречный лазерный пучок. Для описания данного явления вычисляется поляризационный оператор в поле плоской монохроматической волны в старшем порядке по ее амплитуде. Основной целью исследования является сравнение результатов с данными, полученными в рамках приближения локально постоянного поля (locally constant field approximation, LCFA). В работе установлены границы применимости LCFA в случае плосковолнового внешнего поля. Показано, что частота пробного фотона и частота внешнего поля входят в относительную погрешность симметричным образом. Точный учет пространственно-временной зависимости поля за рамками LCFA становится необходимым на масштабе энергий порядка $0.1-1 \mathrm{MeV}$.

Ключевые слова: вакуумное двулучепреломление, квантовая электродинамика, сильные поля, нелинейные эффекты КЭД.

DOI: $10.21883 /$ OS.2021.07.51084.2067-21

\section{1. Введение}

Еще в 30-е годы прошлого столетия выяснилось, что в рамках квантовой теории возникает эффективное взаимодействие между электромагнитными полями за счет вакуумных флуктуаций заряженных фермионов [13]. Например, при наличии сильного фонового электрического поля излучение распространяется так, как будто вакуум становится поляризованной средой. В результате вакуумные флуктуации даже в отсутствие реальных частиц приводят к целому ряду нелинейных явлений, таких как спонтанное рождение электрон-позитронных пар $[2,4,5]$, рассеяние света на свете $[1,6]$, рождение пары в столкновении двух фотонов [7], расщепление фотона [8-10] и др. [11-15]. Настоящее исследование посвящено еще одному нелинейному процессу, так называемому вакуумному двулучепреломлению, при котором происходит изменение поляризации света при прохождении через сильное фоновое поле [9,10,16-18]. Во внешнем поле вакуум ведет себя как двулучепреломляющая среда, т.е. показатель преломления света в вакууме начинает зависеть от поляризации. В частности, это приводит к тому, что часть фотонов линейно поляризованного излучения приобретает поляризацию, ортогональную исходной.

Вакуумное двулучепреломление еще не исследовалось на практике, поскольку напряженность полей, создаваемых в лабораториях, на порядки меньше критических значений $E_{\mathrm{c}}=m^{2} c^{3} /(|e| \hbar) \sim 10^{16} \mathrm{~V} / \mathrm{cm}$ и $B_{\mathrm{c}}=m^{2} c^{2} /(|e| \hbar) \sim 10^{9}$ Т для электрического и магнит- ного полей соответственно ( $m$ и $e-$ масса и заряд электрона соответственно). Данное обстоятельство делает величину эффекта чрезвычайно малой. Одним из возможных экспериментальных сценариев является измерение поляризации света, проходящего через область пространства, в которой создано сильное магнитное поле [10-21]. Кроме того, стремительное развитие лазерных технологий позволяет рассчитывать на то, что в недалеком будущем поворот плоскости поляризации излучения можно будет измерить при столкновении пробных фотонов с лазерным импульсом высокой интенсивности [22-30]. Как правило, предполагается, что в такого рода экспериментах энергия фотонов отвечает рентгеновской области. В этом случае экспериментальная техника позволяет с очень высокой точностью измерить поляризацию. Однако если энергия пробных фотонов существенно превосходит $\mathrm{MeV}$, то сама величина эффекта также значительно возрастает, что может скомпенсировать снижение точности измерений в области высоких энергий [31-33].

С точки зрения теории описание вакуумного двулучепреломления в области энергий, меньших $m c^{2}$, сводится к вычислению фейнмановской диаграммы, изображенной на рис. 1. Сворачивая волновые функции исходного и конечного фотонов с поляризационным тензором, можно вычислить амплитуду такого процесса в зависимости от поляризации пробного фотона и параметров внешнего поля. Главная трудность такого подхода заключается в том, что замкнутые выражения для поляризационного оператора известны лишь в простейших случаях, когда 


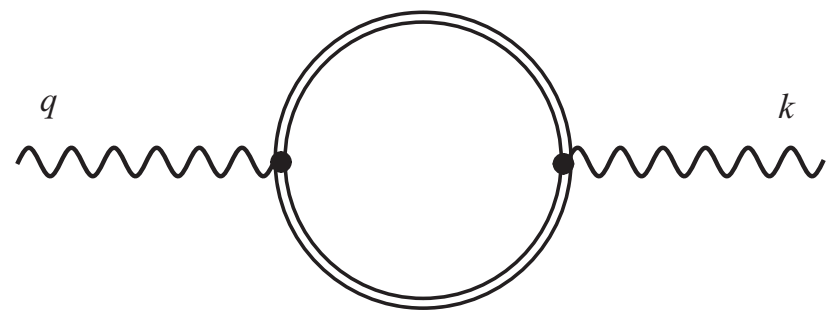

Pис. 1. Диаграмма Фейнмана, описывающая вакуумное двулучепреломление. Двойная линия отвечает точному электронному пропагатору во внешнем поле.

внешнее поле не зависит ни от координат, ни от времени [11,34-37] или представляет собой плоскую электромагнитную волну [38-40]. Возможность анализировать эффект в неоднородных полях произвольной геометрии возникает при использовании так называемого приближения локально постоянного поля (locally constant field approximation, LCFA). Идея этого подхода заключается в том, что выражение для поляризационного тензора, полученное для постоянного поля, представляется в виде интеграла по пространственно-временным координатам, где затем используются реальные значения компонент напряженности внешнего поля. Таким образом, поле считается локально постоянным, что справедливо при достаточно малых частотах, удовлетворяющих условию $\hbar \omega \ll m c^{2}$. Заметим, что данный приближенный подход позволил получить целый ряд важных теоретических предсказаний $[24,27,29,30]$. Применимость LCFA также зависит от энергии пробного фотона. В частности, известно, что при энергиях фотона существенно больше $m c^{2}$ данное приближение использовать нельзя. В рамках данного исследования мы проанализируем, как точность LCFA зависит от частоты лазерного поля и пробных фотонов, при помощи сравнения предсказаний LCFA c результатами точных вычислений фейнмановской диаграммы, представленной на рис. 1, в простейшем случае, когда фотон распространяется в поле плоской монохроматической волны. Несмотря на то, что для такой конфигурации внешнего поля существует аналитическое выражение для поляризационного оператора, мы будем вычислять диаграмму напрямую по теории возмущений, поскольку такой подход может быть эффективно обобщен для рассмотрения более сложных сценариев.

В работе использована релятивистская система единиц $(\hbar=1, \quad c=1)$ и единицы заряда $\alpha=e^{2} / 4 \pi$ $(\alpha \approx 1 / 137, e<0)$. Метрический тензор выбран в виде $\eta_{\mu v}=\operatorname{diag}(+1,-1,-1,-1)$.

\section{2. Приближение локально постоянного поля}

Диаграмма Фейнмана, изображенная на рис. 1, содержит двойную линию, которая отвечает электронному пропагатору, учитывающему взаимодействие с внешним полем сразу во всех порядках. Поскольку амплитуда лазерного импульса $E_{0}$ на практике существенно меньше $E_{\mathrm{c}}$, имеет смысл оценивать эффект в старшем порядке $\left(E_{0} / E_{\mathrm{c}}\right)^{2}$. Пусть $q$ и $k$ отвечают начальному и конечному 4-импульсам фотона соответственно, а внешнее поле, описываемое 4-потенциалом $\mathcal{A}$, удовлетворяет соотношениям $\mathbf{E B}=0$ и $|\mathbf{E}|=|\mathbf{B}|$. Как было показано в [41] (см. также $[27,29,30])$, амплитуда процесса может быть вычислена по формуле

$$
\begin{aligned}
\mathcal{S} & =\frac{1}{(2 \pi)^{3 / 2}} \frac{1}{\sqrt{2 k^{0}}} \varepsilon_{\mu}^{*}(k) \Pi^{\mu \nu}(k, q \mid \mathcal{A}) \\
& \times \frac{1}{(2 \pi)^{3 / 2}} \frac{1}{\sqrt{2 q^{0}}} \varepsilon_{v}(q),
\end{aligned}
$$

где поляризационный тензор имеет вид

$$
\begin{aligned}
& \Pi^{\mu \nu}(k, q \mid \mathcal{A})=-\frac{\alpha}{45 \pi} \frac{e^{2}}{m^{4}} \int d^{4} x \mathrm{e}^{i(k-q) x} \\
& \times\left[4(q F)^{\mu}(k F)^{\nu}+7(q G)^{\mu}(k G)^{\nu}\right] .
\end{aligned}
$$

Здесь $k x \equiv k^{\mu} x_{\mu}$ и $(k F)^{\mu} \equiv k_{\rho} F^{\rho \mu}$. Тензор электромагнитного поля $F_{\mu \nu}=\partial_{\mu} \mathcal{A}_{\nu}-\partial_{\nu} \mathcal{A}_{\mu}$, а также дуальный тензор $G^{\mu \nu}=(1 / 2) \varepsilon^{\mu \nu \rho \sigma} F_{\rho \sigma}$ вычисляются в точке $x$ в соответствии с LCFA. Амплитуда (1) зависит от векторов $q$ и $k$, поляризаций $\varepsilon(k)$ и $\varepsilon(q)$, а также от геометрии и параметров внешнего поля. Заметим, что в выражении (1) для волновых функций фотона мы берем нормировочные факторы $1 /(2 \pi)^{3 / 2}$, которые не использовались в работах $[27,29,30]$.

Мы считаем, что внешнее поле является плоской монохроматической волной, распространяющейся вдоль оси $z$ и поляризованной вдоль оси $x$. Таким образом, поле можно описывать при помощи следующего потенциала:

$$
\mathcal{A}_{x}(t, z)=\frac{E_{0}}{\omega} \sin (\omega t-\omega z)
$$

Остальные компоненты равны нулю. Ненулевые компоненты электрического и магнитного полей имеют вид $E_{x}=B_{y}=-E_{0} \cos (\omega t-\omega z)$.

Направим начальный импульс фотона $\mathbf{q}$ против оси $z$, т.е. против направления распространения лазерного излучения: $\mathbf{q}=\left(0,0,-q^{0}\right)$. При вычислении интегралов по времени и по $z$-координате в выражении (2) будут возникать $\delta$-функции, связывающие величины $k^{0}$ и $k_{z} \mathrm{c}$ $q^{0}$ и $q_{z}$ соответственно. При этом импульс фотона может остаться прежним, а может измениться на величину $\pm 2 \omega$. В последнем случае вероятность такого процесса будет значительно меньше [27], так что мы сосредоточимся лишь на вкладе с $k_{z}=k^{0}=q_{z}=q^{0}$. Результат можно записать в виде

$$
\mathcal{S}=-\frac{k^{0}}{(2 \pi)^{3}} \frac{E_{0}^{2}}{E_{\mathrm{c}}^{2}} \delta\left(k^{0}-q^{0}\right) \delta(\mathbf{k}-\mathbf{q}) \varepsilon_{\mu}^{*}(k) \mathfrak{J}^{\mu \nu} \varepsilon_{v}(q),
$$



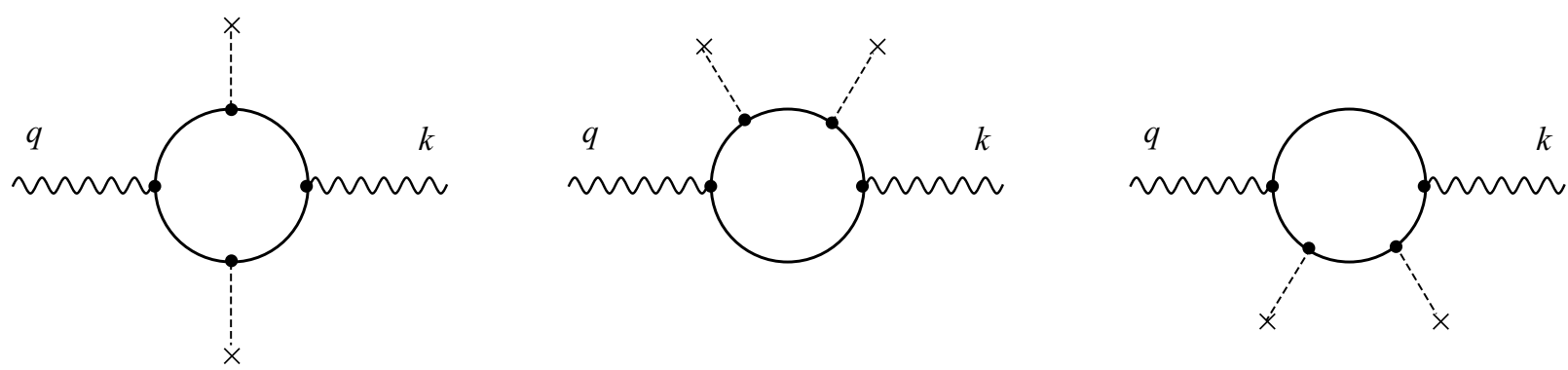

Pис. 2. Диаграммы Фейнмана, описывающие вакуумное двулучепреломление во втором порядке по $E_{0} / E_{\mathrm{c}}$.

который будет также справедлив в случае точного расчета. В рамках LCFA матрица $y^{\mu v}$ дается безразмерным выражением

$$
y_{\text {LCFA }}^{\mu \nu}=\frac{16 \pi^{3} \alpha}{45}\left(\begin{array}{cccc}
0 & 0 & 0 & 0 \\
0 & 4 & 0 & 0 \\
0 & 0 & 7 & 0 \\
0 & 0 & 0 & 0
\end{array}\right)
$$

Отличие двух диагональных элементов этой матрицы как раз свидетельствует о зависимости коэффициента преломления от поляризации света, что может приводить к изменению поляризации пробных фотонов. Мы видим, что амплитуда (4) пропорциональна $k^{0}$ и никак не зависит от частоты внешнего поля $\omega$. Как будет показано ниже, точный результат за рамками LCFA для $y^{\mu v}$ приобретает зависимость как от $k^{0}$, так и от $\omega$.

\section{3. Расчет за рамками LCFA}

Для нахождения интересующей нас амплитуды в старшем (втором) порядке по параметру $E_{0} / E_{\mathrm{c}}$ требуется вычислить сумму трех фейнмановских диаграмм, представленных на рис. 2. Штриховая линия с крестиком обозначает взаимодействие с внешним классическим полем. Поскольку поле считается монохроматическим, каждая вершина взаимодействия отвечает изменению импульса на $\pm \omega$. В результате переданный импульс $k_{z}-q_{z}$ может принимать значения $0,-2 \omega$ и $+2 \omega$, как и было предсказано LCFA. Заметим также, что вычисление данных диаграмм похоже на расчет вкладов в вакуумное излучение фотонов [42-47].

Выпишем в качестве примера вклад в $\mathscr{Y}^{\mu \nu}$ от первой диаграммы на рис. 2 для случая нулевого переданного импульса $(k=q)$ :

$$
\begin{aligned}
& \mathscr{Y}_{(1)}^{\mu \nu}\left(k^{0}, \omega\right)=\frac{\pi \alpha}{2} \frac{m^{2}}{k_{0}^{2}} \frac{m^{2}}{\omega^{2}} \sum_{\beta= \pm 1} \int_{C_{\mathrm{F}}} d \tilde{\omega} \int d \mathbf{p} \\
& \times \operatorname{Tr}\left[\gamma^{\mu} S(\mathbf{p}, \tilde{\omega}) \gamma^{1} S(\mathbf{p}+\beta \mathbf{K}, \tilde{\omega}+\beta \omega) \gamma^{\nu}\right. \\
& \left.\times S\left(\mathbf{p}-\mathbf{k}+\beta \mathbf{K}, \tilde{\omega}-k^{0}+\beta \omega\right) \gamma^{1} S\left(\mathbf{p}-\mathbf{k}, \tilde{\omega}-k^{0}\right)\right]
\end{aligned}
$$

где

$$
S(\mathbf{p}, \tilde{\omega})=\frac{\gamma^{0} \tilde{\omega}-\gamma \mathbf{p}+m}{m^{2}+\mathbf{p}^{2}-\tilde{\omega}^{2}},
$$

$\gamma^{\mu}$ - матрицы Дирака и $\mathbf{K}=(0,0, \omega)$. Интегрирование по $\tilde{\omega}$ в (6) производится по стандартному контуру для причинной функции Грина, который в численных расчетах мы разворачиваем вдоль мнимой оси. Интеграл по p также находится численно. Физический смысл имеет квадрат модуля амплитуды, так что результаты будут представлены в терминах $\left|Y^{\mu v}\right|^{2}$. В рамках точного подхода к выражению (6) необходимо добавить вклады от двух других диаграмм на рис. 2 и вычислить квадрат модуля получившейся суммы.

Далее мы проанализируем точность LCFA в зависимости от параметров $k^{0}$ и $\omega$.

\section{4. Численные результаты и обсуждение}

Для количественного сравнения предсказаний LCFA с точными результатами в старшем порядке по $E_{0} / E_{\mathrm{c}}$ сосредоточимся на элементе $\mathscr{Y}^{11}$. Имеем

$$
y_{\mathrm{LCFA}}^{11}=\frac{64 \pi^{3} \alpha}{45}, \quad\left|\mathscr{Y}_{\mathrm{LCFA}}^{11}\right|^{2} \approx 0.104
$$

Можно явно показать, что квадрат амплитуды, вычисленный с помощью диаграмм, т.е. без использования LCFA, обладает следующим свойством:

$$
\left|\mathcal{I}^{11}\left(k^{0}, \omega\right)\right|^{2}=\left|\mathcal{I}^{11}\left(\omega, k^{0}\right)\right|^{2} .
$$

Если ввести относительную погрешность, связанную с использованием LCFA,

$$
\Delta\left(k^{0}, \omega\right)=\frac{\left.|| Y^{11}\left(k^{0}, \omega\right)\right|^{2}-\left|\mathscr{Y}_{\text {LCFA }}^{11}\right|^{2} \mid}{\left|\mathscr{Y}^{11}\left(k^{0}, \omega\right)\right|^{2}},
$$

то она также будет симметрична: $\Delta\left(k^{0}, \omega\right)=\Delta\left(\omega, k^{0}\right)$. Это означает, что на применимость LCFA и точность приближенных результатов в равной степени влияет выбор частоты лазера и частоты пробного фотона. Важно отметить, что квадрат модуля амплитуды $|\mathcal{S}|^{2}$ не является симметричным по $k^{0}$ и $\omega$, но относительная погрешность в вычислении $|\mathcal{S}|^{2}$ также равна $\Delta\left(k^{0}, \omega\right)$. 


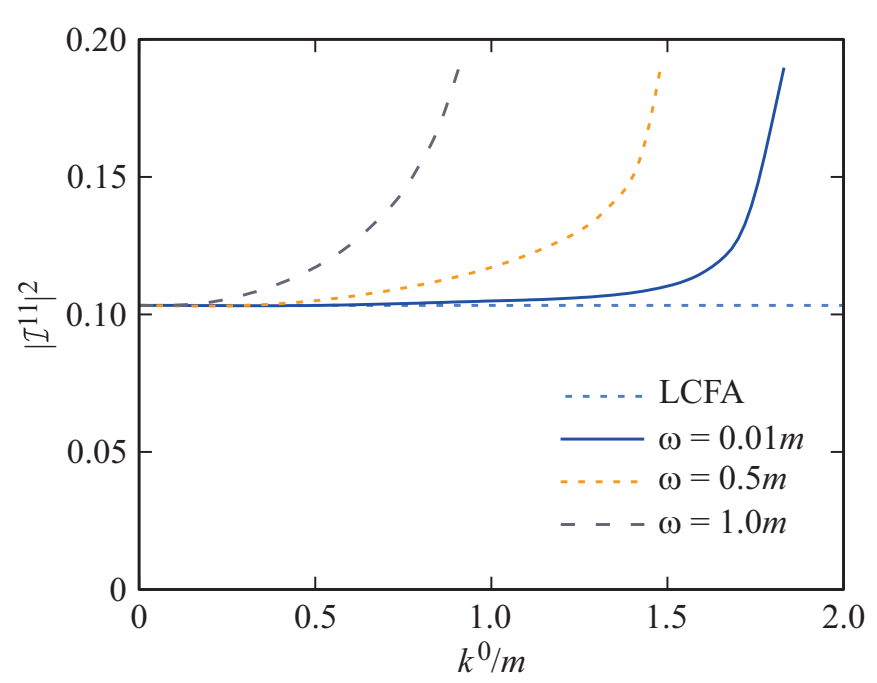

Рис. 3. Зависимость $\left|y^{11}\right|^{2}$ от частоты пробного фотона $k^{0}$ для различных значений частоты внешнего поля $\omega$, полученная при вычислении фейнмановских диаграмм, изображенных на рис. 2, а также результат (8), полученный в рамках LCFA (горизонтальная штриховая линия).

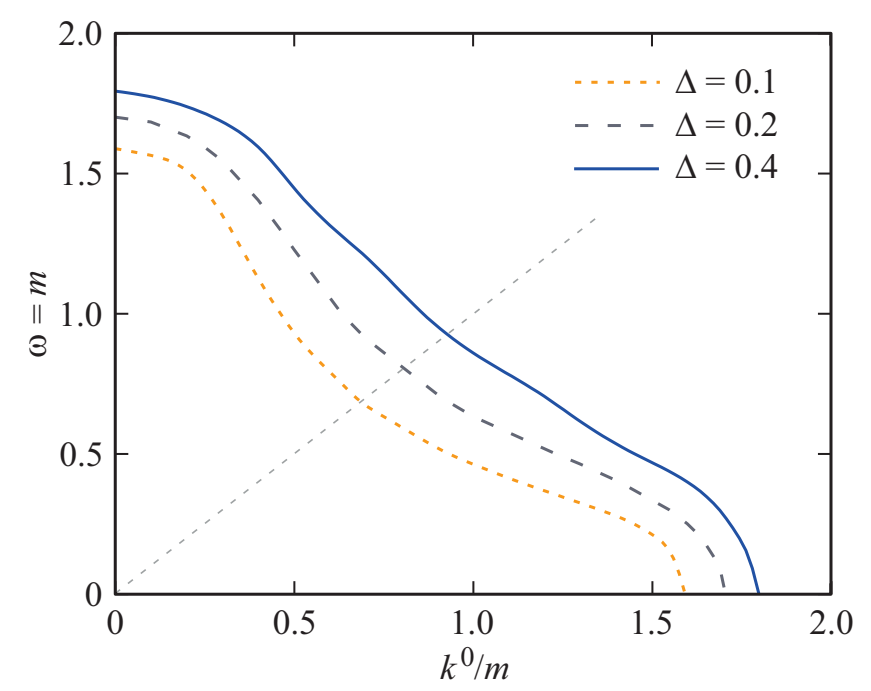

Рис. 4. Линии уровня функции $\Delta\left(k^{0}, \omega\right)$ на плоскости $k^{0}-\omega$, отвечающие относительной погрешности LCFA в 10, 20 и 40\%. Кривые симметричны относительно серой штриховой линии, исходящей из начала координат и являющейся биссектрисой $\omega=k^{0}$.

На рис. 3 представлена зависимость величины $\left|y^{11}\right|^{2}$ от $k^{0}$ для различных значений $\omega$. В соответствии с (8) результат, полученный в рамках LCFA, никак не зависит от $k^{0}$ и $\omega$. Мы видим, что при достаточно высоких частотах $k^{0}$ точные значения оказываются заметно больше, чем приближенные предсказания, полученные в рамках LCFA. При этом чем больше становится $\omega$, тем раньше возникает соответствующее расхождение. Вычислим далее относительную погрешность $\Delta\left(k^{0}, \omega\right)$ для различных значений частот внешнего поля и пробного фотона.
На рис. 4 изображены линии уровня функции $\Delta\left(k^{0}, \omega\right)$ на плоскости $k^{0}-\omega$, которые соответствуют относительной погрешности в 10, 20 и 40\%. Представленные кривые симметричны относительно биссектрисы $\omega=k^{0}$. Данный график позволяет количественно оценить, можно ли использовать LCFA для тех или иных параметров. Например, при $\omega=k^{0}=0.5 m$, т. е. при энергиях порядка $0.2 \mathrm{MeV}$, погрешность составляет примерно $2 \%$, а для того же значения $\omega$ и $k^{0}=m$ она становится равной $12 \%$. Заметим, что характерная частота современных лазеров высокой интенсивности в наших единицах имеет порядок $10^{-6} m$. Это означает, что применимость LCFA определяется энергией пробных фотонов, которая в соответствии с нашими результатами может достигать значений порядка $1.0 \mathrm{~m}$ с сохранением погрешности не более $2 \%$. Таким образом, LCFA позволяет получать надежные предсказания, касающиеся экспериментов с участием лазера и пробных фотонов рентгеновской частоты, поскольку их энергия не превосходит уровня $500 \mathrm{keV}[27,29,30]$.

C другой стороны, одним из возможных экспериментальных сценариев для исследования вакуумного двулучепреломления также является столкновение лазерного импульса высокой интенсивности с гаммаквантом, энергия которого может значительно превышать $1 \mathrm{MeV}$ [31-33]. Для теоретического описания таких процессов требуется выход за рамки LCFA.

\section{5. Заключение}

Вакуумное двулучепреломление в поле плоской монохроматической волны исследовано в старшем порядке по амплитуде внешнего поля. Было проведено количественное сравнение точных результатов с приближенными данными, полученными с использованием приближения локально постоянного поля. Погрешность, связанная с применением LCFA, была вычислена для различных значений частоты внешнего поля $\omega$ и энергии пробного фотона $k^{0}$. Было показано, что эти величины входят в погрешность симметричным образом. В частности, это означает, что при $\omega \ll k^{0}$ относительная величина ошибки полностью определяется энергией фотона $k^{0}$. В этом случае, чтобы погрешность не превосходила $2 \%$, требуется следить за тем, чтобы параметр $k^{0}$ был меньше величины порядка $500 \mathrm{keV}$.

В соответствии с полученными результатами LCFA можно использовать для теоретического описания вакуумного двулучепреломления при использовании фотонов, касающиеся экспериментов с участием лазера и пробных фотонов рентгеновской частоты. Однако возможные сценарии с участием гамма-квантов высокой энергии (например, энергии порядка 10-1000 MeV [31-33]) требуется анализировать за рамками LCFA, т.е. с точным учетом пространственновременной зависимости внешнего поля. 


\section{Финансирование работы}

Исследование выполнено при финансовой поддержке РФФИ и Госкорпорации „Росатом“ в рамках научного проекта № 20-21-00098.

\section{Конфликт интересов}

Авторы заявляют, что у них нет конфликта интересов.

\section{Список литературы}

[1] Euler H., Kockel B.// Naturwissenschaften. 1935. V. 23. P. 246.

[2] Heisenberg W., Euler H. // Z. Phys. 1936. V. 98. P. 714.

[3] Weisskopf V. // K. Dan. Vidensk. Selsk., Mat. Fys. Medd. 1936. V. XIV. P. 6.

[4] Sauter F. // Z. Phys. 1931. V. 69. P. 742.

[5] Schwinger J. // Phys. Rev. 1951. V. 82. P. 664.

[6] Karplus R., Neuman M. // Phys. Rev. 1951. V. 83. P. 776.

[7] Breit G., Wheeler J.A. // Phys. Rev. 1934. V. 46. P. 1087.

[8] Adler S.L., Bahcall J.N., Callan C.G., Rosenbluth M.N. // Phys. Rev. Lett. 1970. V. 25. P. 1061.

[9] Bialynicka-Birula Z., Bialynicki-Birula I. // Phys. Rev. D. 1970. V. 2. P. 2341.

[10] Adler S.L. // Ann. Phys. 1971. V. 67. P. 599.

[11] Dittrich W., Gies H. // Springer Tracts Mod. Phys. 2000. V. 166. P. 1.

[12] Marklund M., Lundin J. // Eur. Phys. J. D. 2009. V. 55. P. 319.

[13] Dunne G. // Eur. Phys. J. D. 2009. V. 55. P. 327.

[14] Heinzl T., Ilderton A. // Eur. Phys. J. D. 2009. V. 55. P. 359.

[15] Di Piazza A., Müller C., Hatsagortsyan K.Z., Keitel C.H. // Rev. Mod. Phys. 2012. V. 84. P. 1177.

[16] Toll J.S. Ph. D. Thesis. Princeton University, 1952.

[17] Baier R., Breitenlohner P. // Acta Phys. Austriaca. 1967. V. 25. P. 212.

[18] Baier R., Breitenlohner P. // Nuovo Cimento B. 1967. V. 47. P. 117.

[19] Cadène A., Berceau P., Fouché M., Battesti R., Rizzo C. // Eur. Phys. J. D. 2014. V. 68. P. 16.

[20] Della Valle F., Ejlli A., Gastaldi U., Messineo G., Milotti E., Pengo R., Ruoso G., Zavattini G. // Eur. Phys. J. C. 2016. V. 76. P. 24

[21] Fan X., Kamioka S., Inada T., Yamazaki T., Namba T., Asai S., Omachi J., Yoshioka K., Kuwata-Gonokami M., Matsuo A., Kawaguchi K., Kindo K., Nojiri H. // Eur. Phys. J. D. 2017. V. 71. P. 308.

[22] Александров Е.Б., Ансельм А.А., Москалев А.Н. // ЖЭТФ. 1985. T. 89. C. 1181.

[23] Heinzl T., Liesfeld B., Amthor K.U., Schwoerer H., Sauerbrey R., Wipf A. // Opt. Commun. 2006. V. 267. P. 318.

[24] Di Piazza A., Hatsagortsyan K.Z., Keitel C.H. // Phys. Rev. Lett. 2006. V. 97. P. 083603.

[25] Dinu V., Heinzl T., Ilderton A., Marklund M., Torgrimsson G. // Phys. Rev. D. 2014. V. 89. P. 125003.

[26] Dinu V., Heinzl T., Ilderton A., Marklund M., Torgrimsson G. // Phys. Rev. D. 2014. V. 90. P. 045025.

[27] Karbstein F., Gies H., Reuter M., Zepf M. // Phys. Rev. D. 2015. V. 92. P. 071301

[28] King B., Heinzl T. // High Power Laser Sci. Eng. 2016. V. 4. P. e5.
[29] Karbstein F., Sundqvist C. // Phys. Rev. D. 2016. V. 94. P. 013004.

[30] Karbstein F. // Phys. Rev. D. 2018. V. 98. P. 056010.

[31] King B., Elkina N. // Phys. Rev. A. 2016. V. 94. P. 062102.

[32] Nakamiya Y., Homma K. // Phys. Rev. D. 2017. V. 96. P. 053002.

[33] Bragin S., Meuren S., Keitel C.H., Di Piazza A. // Phys. Rev. Lett. 2017. V. 119. P. 250403.

[34] Баталин И.А., Шабад А. Е. // ЖЭТФ. 1971. Т. 60. С. 894.

[35] Байер В.Н., Катков В.М., Страховенко В.М. // ЖЭТФ. 1975. T. 68. C. 405.

[36] Urrutia L.F. // Phys. Rev. D. 1978. V. 17. P. 1977.

[37] Schubert C. // Nucl. Phys. B. 2000. V. 585. P. 407.

[38] Байер В.Н., Мильштейн А.И., Страховенко В.М. // ЖЭТФ. 1975. Т. 69. С. 1893.

[39] Becker W., Mitter H. // J. Phys. A. 1975. V. 8. P. 1638.

[40] Meuren S., Keitel C.H., Di Piazza A. // Phys. Rev. Lett. 2013. V. 88. P. 013007.

[41] Karbstein F., Shaisultanov R. // Phys. Rev. D. 2015. V. 91. P. 085027.

[42] Karbstein F., Shaisultanov R. // Phys. Rev. D. 2015. V. 91. P. 113002.

[43] Aleksandrov I.A., Plunien G., Shabaev V.M. // Phys. Rev. D. 2019. V. 100. P. 116003.

[44] Di Piazza A., Hatsagortsyan K.Z., Keitel C.H. // Phys. Rev. D. 2005. V. 72. P. 085005.

[45] Fedotov A.M., Narozhny N.B. // Phys. Lett. A. 2007. V. 362. P. 1.

[46] Narozhny N.B., Fedotov A.M. // Laser Phys. 2007. V. 17. P. 350 .

[47] Aleksandrov I.A., Panferov A.D., Smolyansky S.A. // arXiv:2101.03507. 